New type of wavel et-based spectral anal ysi s by whi ch modes wi th di fferent tor oi dal mode nunber are separ at ed

\begin{tabular}{|l|l|}
\hline $\begin{array}{l}\text { j our nal or } \\
\text { publ i cat } \mathrm{i} \text { on } \mathrm{t} \text { i t l e }\end{array}$ & Revi ew of Sci ent i f i c I nst r ument s \\
\hline vol une & 87 \\
\hline number & 11 \\
\hline page $\mathrm{r}$ ange & $11 \mathrm{D} 411$ \\
\hline year & 2016-11-14 \\
\hline URL & ht t p: //hdl . handl e. net /10655/00012772 \\
\hline
\end{tabular}




\title{
New type of wavelet-based spectral analysis by which modes with different toroidal mode number are separated ${ }^{\text {a) }}$
}

\author{
S. Ohdachi ${ }^{1,2}$ \\ ${ }^{1}$ National Institute for Fusion Science, 322-6 Oroshi-cho, Toki 509-5292, Japan \\ ${ }^{2}$ SOKENDAI (The Graduate University for Advanced Studies), 322-6 Oroshi-cho, Toki 509-5292, Japan
}

(Presented XXXXX; received XXXXX; accepted XXXXX; published online XXXXX)

(Dates appearing here are provided by the Editorial Office)

\begin{abstract}
A new type of wavelet-based analysis for the magnetic fluctuations by which toroidal mode number can be resolved is proposed. By using a wavelet, having a different phase toroidally, a spectrogram with a specific toroidal mode number can be obtained. When this analysis is applied to the measurement of the fluctuations observed in the Large Helical Device, MHD activities having similar frequency in the laboratory frame can be separated from the difference of the toroidal mode number. It is useful for the non-stationary MHD activity. This method is usable when the toroidal magnetic probes are not symmetrically distributed.
\end{abstract}

\section{Introduction}

MHD instabilities and their effects on the plasma performance is one of the most important issues to understand for the realization of the magnetically confined nuclear fusion. Magnetic probe, usually referred to as Mirnov coil, provides essential information about the MHD instabilities, such as the frequency and the mode number. Since the magnetic probes measure the magnetic fluctuation from outside the plasma, information from different sources, which are usually localized at the mode rational surfaces, are superimposed. The most basic analysis for MHD activities is the mode number estimate ${ }^{1}$. Different MHD modes are separated by the peak in the frequency spectrum and mode number is estimated by using the relative phase measured by the probes distributed toroidally and poloidally. In the Large Helical Device (LHD), the magnetic shear in the peripheral region is large. Since there are several rational surfaces and interchange modes are unstable there ${ }^{2}$, several coherent modes related with those rational surfaces are simultaneously observed. The peaks in the frequency spectrum are sometimes overlapped and not easily separated. Another difficulty comes from the fact that the plasma is not stationary. If the MHD instabilities are stationary ones, simple Fourier transform based analysis is sufficient. However, when the MHD activities evolve and/or the frequency is changing, the stationary assumption breaks. Fourier transform in short period is routinely used to obtain time-frequency spectrum or spectrogram. However, when the time scale of the change in the MHD activities is equivalent to or shorter than one time window of the Fourier transform, estimation of the time evolution is quite doubtful. Fourier transform in space ${ }^{3}$ has been used in experiments, as well. Time evolution of the power of the mode having a specific mode number can be obtained. If we assume the wave is a single propagating wave as $f(x)=a \sin (-\mathrm{i} \omega t-n \phi)$, where $\phi$ is the toroidal angle and $n$ is the toroidal mode number, the measured signal at the location of i-th probe located at $\phi=\phi_{\mathrm{i}}$ should bea $\sin \left(-\mathrm{i} \omega t-n \phi_{i}\right)$. Summation with phase information gives $\left|\sum_{i=1}^{N} f(x) \exp \left(-i n \phi_{i}\right)\right|=a(n)$. Amplitude of a mode having the mode number $n$ can thus be estimated. This procedure is simple but quite useful when the distribution of the probes is symmetric and the response of the probe is uniform. In the LHD device of which toroidal period number is 10 , we can put 10 sets of toroidal probes. However, only 6 probes are installed ${ }^{4}$ as shown in Fig. 1. This direct Fourier transform procedure induces large errors in the LHD case. Singular value decomposition of the multi-channel data ${ }^{5}$ is another promising method. However, the separation is not always satisfactory when the magnetic probe distribution is not symmetric and several coherent modes are simultaneously destabilized. In this article, new type analysis in which the different coherent mode is distinguished both by the frequency and by the mode number (e.g. toroidal mode number) is proposed. The separation of the mode is better than the method using only frequency or mode number. The spectrogram of specific toroidal mode can be obtained by this method.

\section{Procedure to distinguish different coherent modes.}

A kind of correlation calculation is proposed to select a coherent mode having a certain frequency and a certain mode number. The correlation of signal to the fragment of waves which have phase difference toroidally, typical shapes of which are shown in Fig. 2(B)-(D), is calculated. Only when the frequency, the toroidal mode number, and the rotation direction is matched finite correlation is detected as shown in Fig. 2(A). The fragment of the wave is basically the Gabor's wavelet whose phase is shifted by its location. The mother wavelet designed for the signal at i-th probe $f_{i}\left(t, \phi_{i}\right)$ is defined as $\psi\left(x, \phi_{i}\right)=$ $C \exp \left(-\frac{x^{2}}{\sigma^{2}}\right) \exp \left(-i x-n \phi_{i}\right)$. Here, $\sigma$ is the parameter to control the locality of the wavelet and $n$ is the toroidal mode number. Wavelet transformation is then given by WT $f(s, u, n)=\sum_{i=1}^{N_{\text {probe }}} \int_{-\infty}^{\infty} f_{i}\left(t, \phi_{i}\right) \frac{1}{\sqrt{s}} \Psi\left(\frac{t-u}{s}, \phi_{i}\right)$. Here, $N_{\text {probes }}$ is the number of the probes. The wavelet spectra of a specific mode number can be estimated by this procedure. Using the inverse wavelet transform, we can then construct the time evolution of the magnetic fluctuations only containing the specific toroidal mode number. From the numerical test with the

${ }^{a)}$ Contributed paper published as part of the Proceedings of the 21st Topical Conference on High-Temperature Plasma Diagnostics (HTPD 2016) in Madison, Wisconsin, USA. 
LHD's magnetic probe configuration, where there are six toroidal magnetic probes $\left(N_{\text {probes }}=6\right)$ distributed not symmetrically, the separation of the signal is well up to $n=3$ component, and $n=4$ component is marginally separated. Components having unintended (unmatched) mode numbers can be reduced by less than $10 \%$

The resolution of this method in space and time is then examined to evaluate the usefulness of this method. Fig. 3(A) shows the correlation to the modes when we try to match a mode of $f=3 \mathrm{kHz}$ and $n=3$. This diagram shows the sensitivities to the unintended wave having different frequency and mode number. The sensitive region is spreading out from the targeting point of $f$ $=3 \mathrm{kHz}$ and $n=3$. This effect is explained by the following discussion. This correlation operation described above is equivalent to the operation obtaining a two dimensional spectrum $s\left(\omega, t_{0}\right)$ by two dimensional Fourier transform of the measure signal with time and spatial windows as,

$s^{\text {observe }}\left(\omega, t_{0}, n\right)$

$=\sum_{i=0}^{N_{\text {probe }}} \int_{-\infty}^{\infty} f\left(\phi_{i}, t\right) \exp \left(-\mathrm{i} n \phi_{i}\right) \times \exp \left(-\frac{\left(t-t_{0}\right)^{2}}{\sigma^{2}}\right) \exp (-\omega t) d t$

$=\int_{-\infty}^{\infty} \int_{0}^{2 \pi} \delta\left(\phi-\phi_{i}\right) f(\phi, t) \exp \left(-\mathrm{i} n \phi_{i}\right) d \phi \exp \left(-\frac{\left(t-t_{0}\right)^{2}}{\sigma^{2}}\right) \exp (-\omega t) d t$

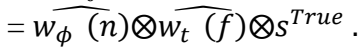

Here, $\otimes$ denotes the convolution. Therefore, it is clear that observed spectrum $s^{\text {observe }}\left(\omega, t_{0}, n\right)$ is blurred spectrum from the true spectrum $s^{\text {True }}$ by the window functions $\widehat{w_{\phi}(n)}$ and $\widehat{w_{t}(f)}$. The cross section of the sensitivity at toroidal mode number $n=3$ and at frequency $f=3 \mathrm{kHz}$ of Fig. 3(A) is shown in Fig. 3(B) and Fig. 3(C), respectively, They are the real part of the window functions of $\widehat{w_{\phi}(n)}$ and $\widehat{w_{t}(f)}$. It is understood that the time / frequency resolution is similar to that of the standard wavelet analysis. With the control of parameter $\sigma$, time / frequency resolution can be adjusted. The spatial resolution depends on the distribution of the location of the probes. When the fluctuations consist of the waves having an integer number, the separation of the mode is good. In paticular if the probes are symmetrically distributed, $\left|\widehat{w_{\phi}(\Delta n)}\right|=0$ is guaranteed, when $\Delta \mathrm{n}$ is integer number (see red line in Fig. 3(B)). When there are missing probes, such as the LHD case, there is finite cross-talk from the unintended coherent mode having integer mode number. However, as shown in Fig. 3(A), distinction is done both by the frequency difference and by the mode number difference. In the experiments, it is not likely that two modes having the same frequency and the same toroidal mode appear simultaneously. Therefore, practically, two coherent modes can be distinguished. This is an advantage of this method.

\section{Example of the spectrogram obtained by this method}

Figure 4(B) shows a spectrogram calculated by the short-time FFT in LHD. H-mode transition is observed at around 3.81s. With the H-mode transition in LHD, strong MHD activity (resistive interchange mode) is activated ${ }^{6}$ from the increase of the pressure gradient just after the transition, shown by the dotted line in Fig. 4. There are rational surfaces of $\iota=1.5$ and 2.0 within the transport barrier. Increase of the $\mathrm{m} / \mathrm{n}=2 / 3,1 / 2$ component is therefore observed. Time around the transition is analyzed by this method as shown in Fig. 5. Rapid increase of the modes of $\mathrm{n}=2(\mathrm{~m} / \mathrm{n}=1 / 2)$ and $\mathrm{n}=3(\mathrm{~m} / \mathrm{n}=2 / 3)$ are clearly caught by this method. It is noted that the MHD instabilities localized at the rational surface in the peripheral region exhibit the bursting nature. The characteristics of the bursting MHD instabilities are studied using the method we proposed in this article in Reference 7.

\section{Summary}

In summary, a new type of wavelet-based analysis, by which time-frequency spectrum having specific toroidal mode number and propagating direction, is proposed. Spatial resolution is determined by the probe arrangement, and frequency/time resolution can be adjusted from the shape of the wavelet. Even though the toroidal alignment of the probe is not symmetric, different modes can be effectively separated since they are separated both by the frequencies and by the mode numbers. It is quite practical in the case when some probes are damaged experimentally. It is useful to study the MHD activities destabilized in the peripheral region of the LHD where the mode frequency overlapped each other. Advanced characteristics of the MHD activities, such as the time interval of the burst, bi-phase of the two MHD activities etc., can be studied systematically using this method.

\section{ACKNOWLEDGMENTS}

The author appreciates very much the valuable comments and the encouragement from the Dr. K. Fujii and Prof. S. Inagaki. He also appreciates the LHD experiment group for the usage of the experimental data. This work was supported by NIFS budget code ULPP021, 028 and is also partially supported by the Ministry of Education, Science, Sports and Culture Grant-in-Aid for Scientific Research 26249144, by the JSPS-NRF-NSFC A3 Foresight Program (NSFC: No.11261140328, NRF: No. 2012K2A2A6000443), and by NIFS/NINS under the project of Formation of International Scientific Base and Network.

${ }^{1}$ E.J. Strait, E.D. Fredrickson, J.-M. Moret, and M. Takechi, Fusion Science and Technology 53, 304 (2008).

${ }^{2}$ K.Y. Watanabe, Y. Suzuki, S. Sakakibara, and others, Fusion Science and Technology 58, 160 (2010).

${ }^{3}$ R.S. Granetz, Review of Scientific Instruments 52, 1332 (1981).

${ }^{4}$ S. Sakakibara, H. Yamada, LHD Experimental Group, Fusion Science and Technology 58, 471 (2010).

${ }^{5}$ C. Nardone, Plasma Physics and Controlled Fusion 34, 1447 (1992).

${ }^{6}$ K. Toi, et. al., Fusion Sci. Tech., 58, 61(2010).

${ }^{7}$ S. Ohdachi, Y. Suzuki, et. al., Nuclear Fusion 55, 93006 (2015).

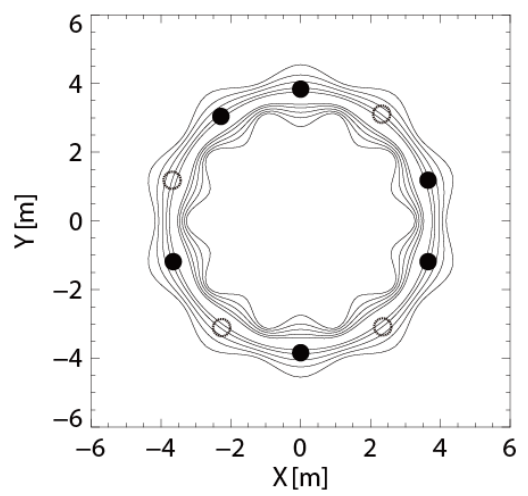

${ }^{a)}$ Contributed paper published as part of the Proceedings of the 21st Topical Conference on High-Temperature Plasma Diagnostics (HTPD 2016) in Madison, Wisconsin, USA. 
FIG. 1. Schematic view of the location of the toroidally distributed magnetic probes on LHD. The locations where the magnetic probes are installed are shown by the closed circle. Flux surfaces of LHD cut at the equatorial plane are also shown by solid lines.

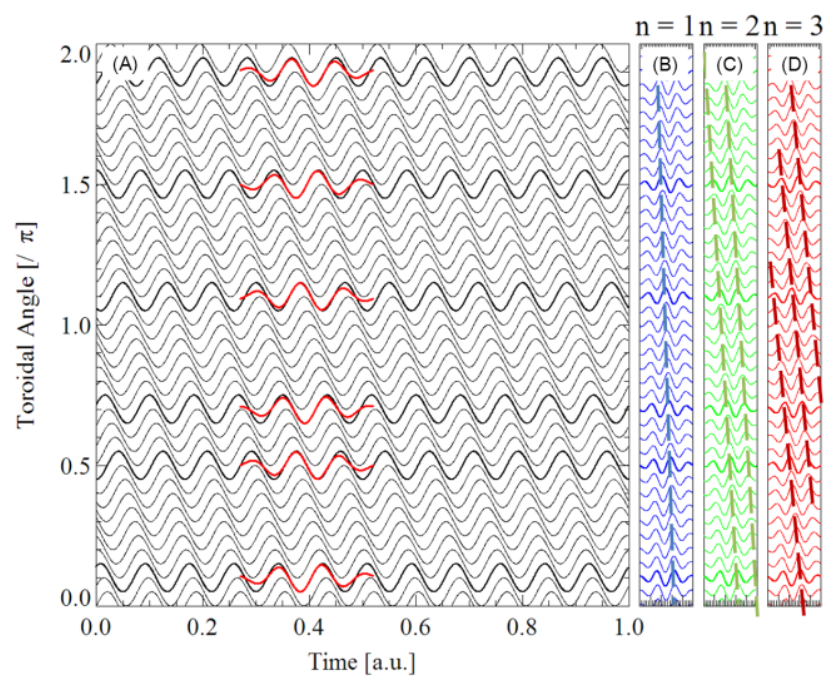

FIG. 2. Schematic diagram of the method. (A) $n=3$ wave is propagating toroidally. (B), (C), and (D) are the wavelets designed for $n=1,2$, and 3 detection, respectively. When $n=3$ mode is to be analyzed, correlation of the wavelet for $n=3$ (D) to the signal is performed within the time window.
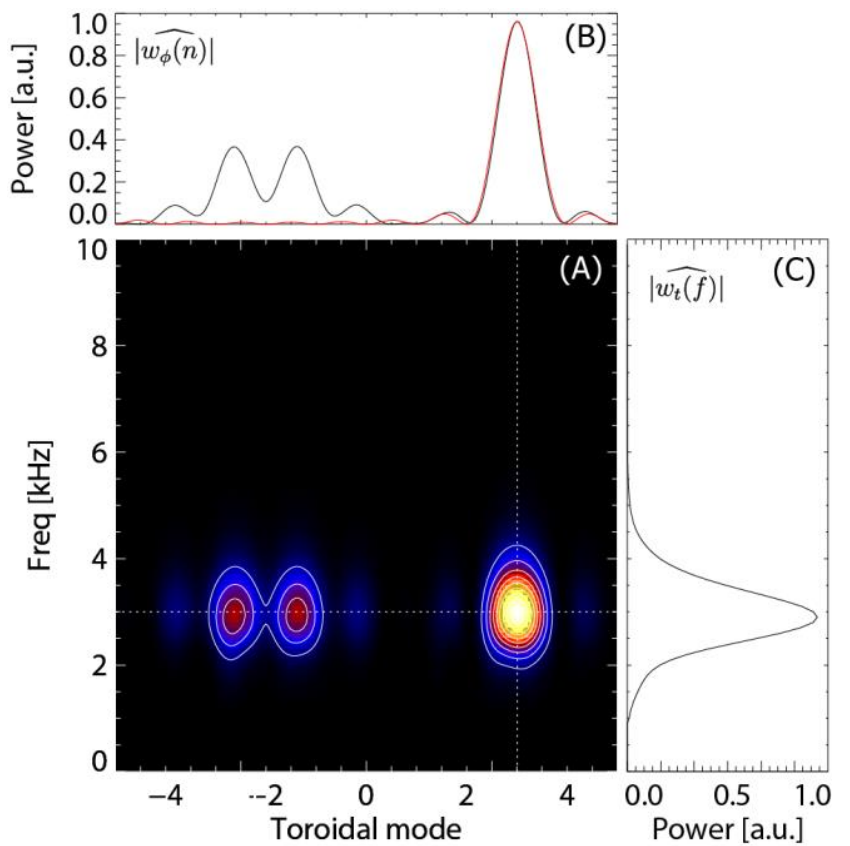

FIG. 3. Correlation to the mode with different toroidal mode number and frequency is shown in (A). Here, detection of the mode with $\mathrm{f}=3 \mathrm{kHz}$ and $\mathrm{n}=3$ is performed with the test waves. Cross section at $\mathrm{n}=3$ and $\mathrm{f}=3 \mathrm{kHz}$, which are equivalent to the window functions, $\widehat{w_{\phi}(n)}$ and $\widehat{w_{t}(f)}$ shown in (B) and (C), respectively. Red line in (B) indicates the case of the 10 probes symmetrically distributed as reference.
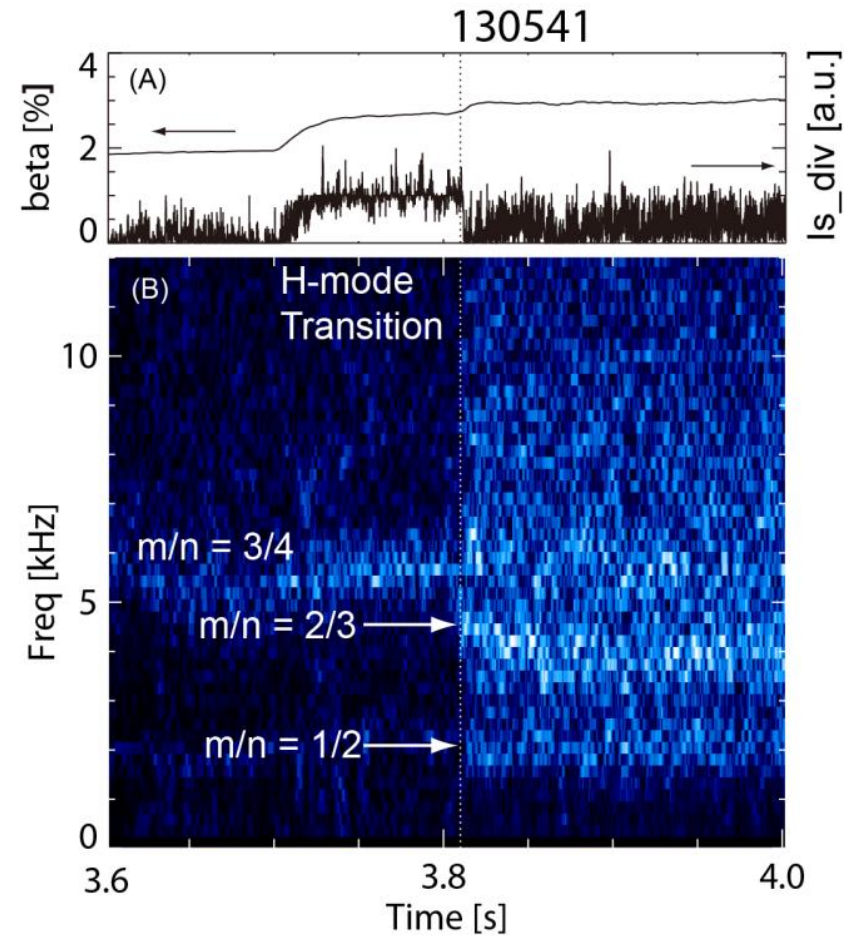

FIG. 4. Time evolution of the volume averaged beta and ion saturation current measured at the divertor plate is shown in (A) and the time-frequency spectrum of the magnetic fluctuations calculated by usual short-time FFT is shown in (B). H-mode transition is observed around 3.81s. Magnetic axis location $R_{\mathrm{ax} 0}=$ $3.56 \mathrm{~m}$ and $\mathrm{B}_{\mathrm{t}}=1.0 \mathrm{~T}$.

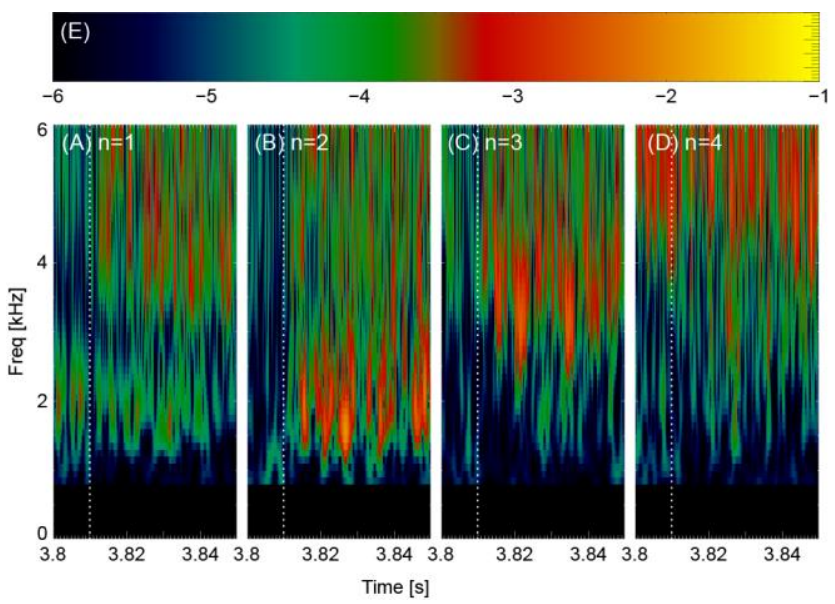

FIG. 5. An example of spectrogram of the $n=1,2,3$, and 4 component are shown in (A), (B), (C), and (D), respectively. Fluctuations below $800 \mathrm{~Hz}$ are not analyzed here due to the long calculation time. The color represents the power of the fluctuation in log-scale shown in (E).

${ }^{a}$ Contributed paper published as part of the Proceedings of the 21st Topical Conference on High-Temperature Plasma Diagnostics (HTPD 2016) in Madison, Wisconsin, USA. 